\title{
Computational Model and Parameter Extraction of High Speed Semiconductor Optical Amplifier Space Switches
}

T. Sutili1 ${ }^{1}$ P. Rocha ${ }^{1}$, Ernesto M. M. Barrientos ${ }^{1,2}$, A. C. Bordonalli ${ }^{1}$, C. M. Gallep ${ }^{3}$, and E. Conforti ${ }^{1}$, ${ }^{1}$ Department of Communications, School of Electrical and Computing Engineering, University of Campinas, Campinas-SP, Brazil

${ }^{2}$ São Paulo Federal Institute (IFSP), Guarulhos, SP, Brazil

${ }^{3}$ School of Technology, University of Campinas, Limeira-SP, Brazil

Corresponding Author: peterson.dmo@gmail.com

\begin{abstract}
A characterization technique, including a mathematical model involving extensive computational resources, is introduced to study the behavior of an electro-optical space switch based on semiconductor optical amplifier (SOA). The model encompasses the precise emulation of device nonlinearities and its transient performance, allowing the characterization of its transient response. The calibration process includes the evaluation of parasites from the SOA connection, mounting and encapsulation, and parameter extraction of the active cavity. The numerical results are compared to experimental data for various operational conditions, with close similarities. The characterization technique can be adapted for other electro-optical devices, such as directedmodulated lasers, amplitude modulators and electro-optical switches.
\end{abstract}

Index Terms-Electro-optical devices, electro-optic switches, equivalent circuit, semiconductor optical amplifier, simulation.

\section{INTRODUCTION}

The exponential increase in fiber optic networks' capacity claimed by new applications such as the Internet of Things, cloud computing, and streaming services calls for novel scientific approaches and low cost technological innovations to fulfill market demands. In this sense, all-optical networks are a promising solution, avoiding the electronic bottlenecks of several optical-electrical-optical (O/E/O) conversions, as well as its inherent latency [1]. In Data Centers (DCs) optical packet switching (OPS) achieves latencies up to $25 \mathrm{~ns}$, representing a clear improvement in comparison with the $90 \mathrm{~ns}$ typically introduced by equivalent all-electrical switches [1]. Complementarily, all-optical interconnects provide power consumption reduction [2], [3], cooling systems simplification and effortless network expansion.

A promising device to achieve integration of complex components in all-optical networks is the semiconductor optical amplifier (SOA). Its versatility permits several electro-optical functions such as WDM amplification [4]]-[[6], wavelength conversion [7], [8], wavelength selection [9], wavelength 
routing [10], and carrier wavelength reusing [11], [12]. Furthermore, its integrability enables the implementation of logic gates [13]-[15], optical adders and subtractors [16], memory modules [17], optical buffers [18]]-[[20], optical demultiplexers [21], and wavelength selectors [22]. Through SOAs nonlinear behavior, it is also possible to project all-optical regenerators for intensity modulated [23], [24], phase encoded [25], [26], and coherent signals [27]. Furthermore, SOAs can perform optical packet switching, allowing the construction of SOA-based OPS $(\mathrm{M} \times \mathrm{N}$ switches), which can establish connections between ports in the order of few nanoseconds [28]. These devices take advantage of the polarization insensitivity [29] and ultrafast transitions from off to on operational states in SOA-based optical space switches [30], which is possible through the dynamic behavior of electrical and optical carriers in the SOA active region [31]]-[[33]. In addition, SOA can simultaneously amplify the switched carrier, compensating for switch insertion loss and improving the optical link signal-to-noise ratio [34].

The study of SOA switching performance shows that the reduction of electrical parasites (especially, capacitances and inductances due to packaging and microwave mounting) results in further improvements in the device electro-optical response velocity and stability. However, there is a trade-off between overshoots and rising times, being all linked to the electrical parasite fading [35]. One tool to evaluate the complex interaction between device parasites and its electro-optical performance is the equivalent circuit-based numerical analysis, which confirmed that packaging inductances and capacitances restrict the switching rise time and induce output power fluctuations [36]. By employing the experimental system response modeling into the electrical equivalent circuit, it is possible to extract important electro-optical parameters such as capacitances, inductances, and resistances inherent to the SOA assembly (transmission and coupling lines, encapsulation, and mounting).

Expanding this approach, the work here proposes the complete modeling of a nonlinear SOA, including the simulation of its microwave parasitic elements and the inference of its constructive and operational properties, which will greatly affect its dynamic performance. Through the comparison of the model behavior and experimental results, it was possible to precisely extract meaningful parameters related to SOA encapsulation, mounting, and architecture. This modeling approach can be applied to any traveling wave SOA (or other electro-optical device), allowing for an analysis of its particular characteristics and performance. Previous works showed that the direct experimental evaluation of these SOA parameters can be challenging, supporting the importance of alternative means to extract this information and emulate it into a precise computational model. In [37], the electric SOA equivalent circuit was used to investigate the mitigation of inter-channel crosstalk by assist light in a TOAD (Terahertz Optical Asymmetric Demultiplexer) switch. The methodology presented in Section II is a new characterization technique based on the employment of extensive 
computational resources in conjunction with experimental references, permitting not only the device modeling but also the analysis of its transient behavior and performance under several operational conditions. As it will be further discussed in Section A, the approach allowed the determination of the electrical microwave pulse signal that is actually injected into the SOA active region, considering distortions caused by electric parasites [35]. By employing the obtained microwave signal as system electrical input, as it will be presented in Section B, it is possible to model the device optical response (i.e., the SOA optical gain and dynamic behavior, as a function of the injected bias current or the optical input power) in relation to its operational and constructive parameters. In [38], the dynamic SOA response for modulated optical signals up to $10 \mathrm{~Gb} / \mathrm{s}$ was obtained by using a so called reservoir model, which adapted parameters previously obtained from a steady-state response model presented in [39]. The dynamic response modeling applied to SOA-based photonic processing, especially using XGM (Cross-Gain Modulation), is presented in [40]. As a matter of fact, SOAs with different characteristics (especially, composition and geometry), will present variations in their optical responses. The results discussed in Section III show the dynamic behavior simulation of a practical optical switch through the computational modelling technique here presented. As addressed in Section $\mathrm{IV}$, this can be a valuable tool to the complete performance characterization of photonic devices in complex optical networks.

\section{MATERIAL AND METHODS}

The SOA dynamic modelling technique is based on two sequential procedures, covering the inference of microwave parasites from the mounting and the device operational and constructive parameter calibration. Firstly, the electrical switching pulse injected into the SOA active region is determined by taking into consideration the transient oscillations induced by the SOA mounting. For this purpose, the SOA experimental optical switching pulses were employed as reference to evaluate each component in a electrical equivalent circuit calibrated through Simulink [41] simulation. In particular, the nonlinear SOA model under analysis and used during the experimental validation is the CIP-NL-OEC-1550, from now on referred to as CIP-NL. Next, the SOA electro-optical dynamic behavior is adjusted employing the mentioned electrical switching pulses as input and, once again, experimental results as reference. Through heuristic extraction employing numerical analysis, it was possible to refine the default VPI [42] SOA model, extracting the devices operational parameters and constructive characteristics and emulating its dynamic performance in experimental conditions.

\section{A Equivalent Circuit Calibration (Simulink)}

By taking advantage of the constructive similarities between SOAs and semiconductor lasers (as a matter of fact, the first is basically the second with anti-reflective coatings in its active region facets), it is possible to adapt well known laser equivalent circuits [43] to simulate the SOA electro-optical 
response. This approach was extensively exploited in previously works [44], [45], [33], resulting in the extraction of approximate parameters from the characterized SOA. However, in those cases, the fitting between simulated equivalent circuit and experimentally evaluated responses was performed only on the frequency-domain, resulting in an inaccurate SOA behavior in time-domain simulations [44]. Furthermore, the equivalent circuit component evaluation was carried out heuristically, resulting in an extensive work to evaluate the complex interaction between all electrical elements. In general, this methodology results in an incomplete characterization of SOA-based switch properties, as evidenced by high-frequency oscillations in time-domain which do not follow the experimental behavior [44]. The proposed methodology is constructed on a different approach that employ the possibility of performing several equivalent circuit simulations directly in time-domain, where electrical component values are varied until the output converges to the desired SOA behavior.

The electrical equivalent circuit is presented in Fig. 1 and contains tree different stages. The first one, named "Coupling", is related to microwave mounting and parasites elements derived from SOA. The second one, referred to as "Encapsulation", is formed by parasitic elements associated with the SOA chip encapsulation in a form factor suitable for commercial distribution. Finally, the third one, called "SOA", emulates the device itself, converting the input electrical microwave pulse in an equivalent optical signal output (proportional to the electrical current $i_{\text {eq }}$ ). A more comprehensive view of the equivalent circuit model is possible through the physical interpretation of each electrical component. Regarding the SOA elements: $C_{\mathrm{S}}$ is a SOA mounting parasitic element dependent on the electrical current injected into the device; $R_{\mathrm{S}}$ stands for a small-loss of the SOA input current in the mounting; $C_{\mathrm{SC}}$ represents the heterojunction space charge capacitance, emulating the electrical carrier storage; $C_{\mathrm{D}}$ is the diffusion capacitance, standing for the electrical carrier lifetime; $R_{\mathrm{E}}$ is the heterojunction effective resistance; $L_{\mathrm{S}}$ is the SOA active region optical carrier storage; $R_{\mathrm{S} 1}$ emulates the SOA gain compression; and, $R_{\mathrm{S} 2}$ represents the device spontaneous emission. However, the electrical current injected into the SOA is previously degraded by the coupling and encapsulation parasitic elements: $T_{\mathrm{L}}$ stands for a transmission line intended to guide the microwave signal into the SOA mounting; $R_{\mathrm{C}}$ is a coupling resistor with matches the mounting input impedance to that of the generator output; $C_{\mathrm{A}}, L_{\mathrm{A}}, C_{\mathrm{B}}$, and $L_{\mathrm{B}}$ are coupling parasitic elements from the microwave connections; $C_{\mathrm{P}}, L_{\mathrm{P}}$, and $R_{\mathrm{P}}$ are parasitic elements linked with the SOA's chip enclosure in its commercial form factor. 


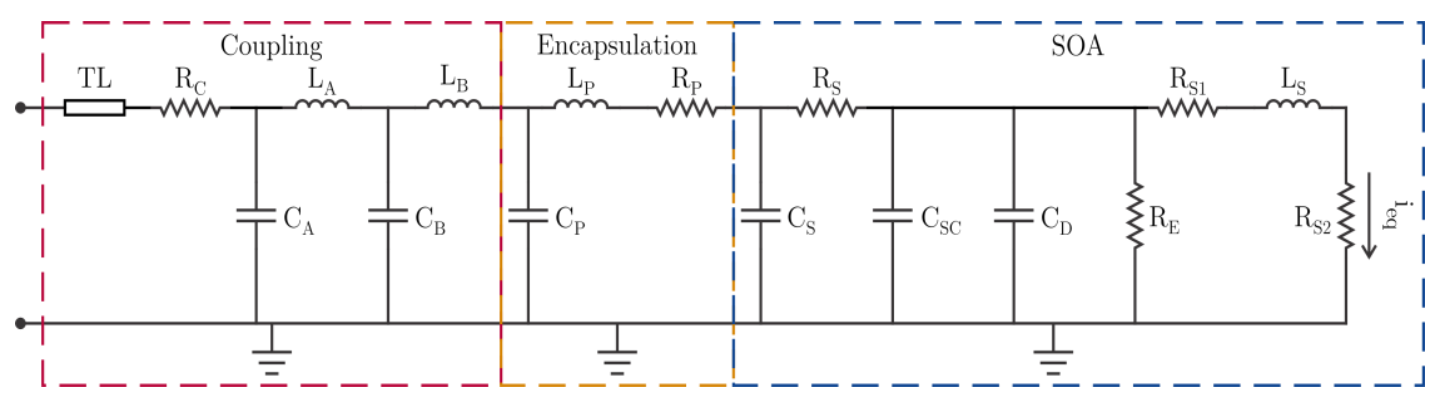

Fig. 1: Equivalent circuit employed in the microwave mounting and SOA active region modelling.

Nevertheless, the SOA is an electro-optical component with inherently nonlinear behavior, characteristic that is especially evident when operating with larger signals, as occurs in the switching process. In this sense, it is impossible for a linear equivalent circuit, as the one proposed in Fig. 1, to perfectly emulate the device dynamic behavior, particularly its transient oscillations after the pulse rising edge. Due to the fundamental limitations of this approach, the equivalent circuit parameters extraction in Simulink environment intended primarily to evaluate the electrical current that is actually injected in the SOA heterojunction. An in-deeper modelling of the SOA itself is carried out in the VPI environment, which has the tools required to carefully adjust the semiconductor constructive and operational characteristics, as discussed in Section B. A better equivalent circuit could then be achieved with nonlinear circuit elements, especially the ones corresponding to the SOA active region emulation. One way to implement that is to adopt current dependent resistors and capacitors, which could model with accuracy the SOA active region refractive index dependence with the device gain and input optical power. Another solution could be the use of time-domain partial linearization of switching pulses, which consist on analysing each pulse segment and considering different electric elements values for each instant, thus matching specifically the SOA operational condition for drastic output power transient oscillations.

Yet, the equivalent circuit was employed here only to approximate the parasitic degradation impacting the electrical current injected into the SOA heterojunction, being appropriate to this purpose. To approximate the equivalent circuit output current $i_{\text {eq }}$ with the actual device behavior, the developed algorithm performs several simulations, adjusting the electrical components values in increasing smaller ranges, getting progressively closer to the experimentally characterized optical switching pulse employed as reference. As each optical component variation has an overall impact in several switching pulse characteristics, it is crucial to constantly evaluate the similarities between the simulated and experimental pulses. With this objective, several evaluation metrics were employed, including pulse rise time, percentage overshoot, transient behavior, stabilization time, percentage undershoot, and mean square error. After each interaction, the developed algorithm automatically evaluates these performance metrics, comparing the circuit output electrical current $i_{\text {eq }}$ (which is equivalent to the SOA optical output power) with experimentally characterized switching pulses. As 
these signals get closer time-domain shapes (evidenced by smaller errors in these metrics), the algorithm saves the equivalent circuit electrical element values, using them as seeds for the next iteration. If one interaction diverges from the expected experimental behavior, the correspondent values are discarded. After several thousand iterations, a good approximation between the equivalent circuit response and the experimental reference is achieved.

The steps followed by the Simulink algorithm are presented in the Fig. 2, showing the three different calibration stages. Initially, the developed algorithm is fed with previously published values for the CIP-NL equivalent circuit [46] as a seed. Aiming a first approximation, only are the parameters with high impact in the switching pulse overall behavior (i.e., $C_{\mathrm{A}}, L_{\mathrm{B}}, L_{\mathrm{P}}$, and $R_{\mathrm{P}}$ ) adjusted in a wide range. After a good initial match is achieved, these values are refined through their variation in smaller steps. Next, a fine tuning of the electrical elements directly linked to the electro-optical conversion (i.e., $R_{\mathrm{S}}, C_{\mathrm{D}}, R_{\mathrm{E}}, R_{\mathrm{S} 1}$, and $L_{\mathrm{S}}$ ) is performed, allowing a final calibration involving all the equivalent circuit electric elements. The results of this approximation and calibration process are illustrated in Fig. 3, where it is possible to note closer approximations between the experimental switching pulse and the equivalent circuit outputs in each stage of Fig. 2.

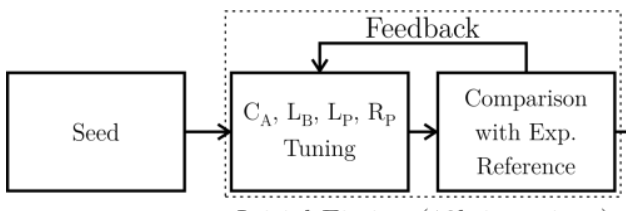

Initial Fitting (10k iterations)

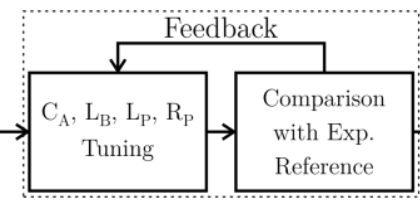

Fine Fitting (4k iterations)

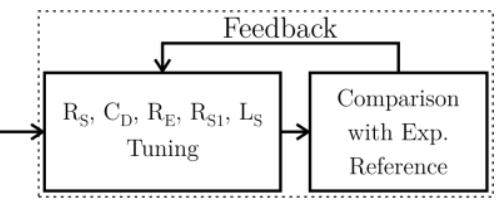

Final Fitting (16k iterations)

Figure 2: Block diagram with the Simulink steps for the equivalent circuit calibration process.

This calibration approach can be quantitatively evaluated through the metrics employed in the developed algorithm, as presented in Table 1, which refers to the pulses in Fig. 3. However, as evidenced by the pulses in this figure, the calibrated equivalent circuit in unable to emulates perfectly the SOA transient behavior. In fact, this behavior is intrinsically linked to its active region nonlinearities and coupled optical and electrical carriers, which cannot be simulated through a linear electrical circuit. As previously discussed, this approach is limited to evaluate the mounting and encapsulation parasitic element impact on the device performance. Therefore, the calibrated equivalent circuit was employed mainly to appraise the electrical current injected into the SOA heterojunction, which was then used in the VPI modelling presented in the next section. 


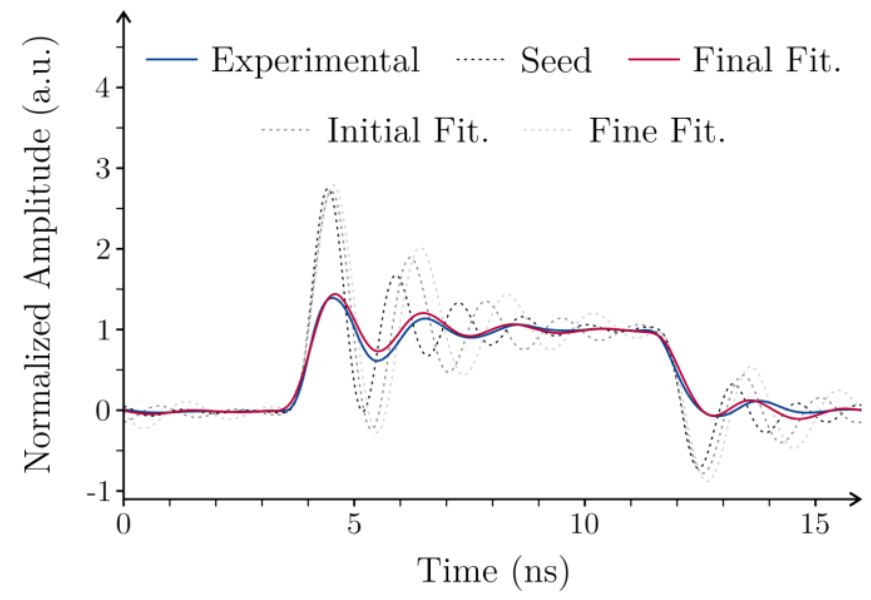

Fig. 3: Time domain switching pulse showing a comparison between experimental and equivalent circuit calibration results for increasingly refinement as a function of the evaluation metrics. The reference experimental pulse was obtained through the switching of a nonlinear SOA (CIP-NL) with $100 \mathrm{~mA}$ bias, $3 \mathrm{~V}$ switching step, and 3 V PISIC pre-impulse.

TABLE I. EQUIVALENT CIRCUIT CALIBRATION METRICS

\begin{tabular}{c|c|c}
\hline Evaluation Metric & Seed Circuit & Calibrated Circuit \\
\hline \hline Rise Time Error & $128.12 \mathrm{ps}$ & $101.56 \mathrm{ps}$ \\
\hline Overshoot Error & $133.67 \%$ & $4.72 \%$ \\
\hline Transient Frequency Error & $314.12 \mathrm{ps}$ & $23.41 \mathrm{ps}$ \\
\hline Stabilization Time Error & $107.78 \mathrm{ps}$ & $31.32 \mathrm{ps}$ \\
\hline Undershoot Error Mean & $63.84 \%$ & $0.41 \%$ \\
\hline Mean Square Error & 1171.49 & 24.88 \\
\hline
\end{tabular}

\section{B Dynamic Response Calibration (VPI)}

The SOA dynamic response calibration was performed following the technique proposed in [47] and detailed in Section C. Through this approach, it was possible to precisely tune the SOA static optical gain in the VPI environment [42] as a function of its bias current and optical input power. This first SOA response approximation was then expanded considering its dynamic behavior when switching pulses, as the ones evaluated in Section A via Simulink environment [41], were summed up with the device bias current, as discussed in Section D.

\section{Static Gain Calibration}

Parameters such as the SOA active region length, width, and thickness can affect the electro-optical switching behavior, since the SOA optical gain is inherently dependent on them. Unfortunately, these parameters are seldom provided by manufacturers, and its experimental extraction is complex. Usually, length and width can be measured using scanning electron microscopy on an open device, and length can be obtained by analysing residual Fabry-Perot modes [48]. However, these parameters, among several others, as showed in Table 2, can be obtained through heuristic extraction, employing numerical analysis, as presented in [47]. 
TABLE II. PARAMETERS FOR SOA MODEL.

\begin{tabular}{cc|cc}
\hline \multicolumn{1}{c}{ Parameter } & Default & Static & Dynamic \\
\hline \hline Active Region Type & MQW & MQW & MQW \\
\hline Width of Active Region & $2.5 \mu \mathrm{m}$ & $650 \mu \mathrm{m}$ & $650 \mu \mathrm{m}$ \\
\hline Cavity Length & $2 \mathrm{~mm}$ & $2 \mathrm{~mm}$ & $2 \mathrm{~mm}$ \\
\hline Thickness of Active Region & $40 \mathrm{~nm}$ & $200 \mathrm{~nm}$ & $200 \mathrm{~nm}$ \\
\hline Thickness of the SCH Region & $210 \mathrm{~nm}$ & $210 \mathrm{~nm}$ & \\
\hline Injection Efficiency Coefficient & 1.00 & 0.85 & 0.85 \\
\hline Internal Losses Cefficient $(I L C)$ & $3000 \mathrm{~m}^{-1}$ & $2500 \mathrm{~m}^{-1}$ & $2500 \mathrm{~m}^{-1}$ \\
\hline Internal Loss Carrier Dependence & $1 \cdot 10^{-23} \mathrm{~m}^{2}$ & $1.1 \cdot 10^{-21} \mathrm{~m}^{2}$ & \\
\hline MQW Confinement Factor & 0.07 & 0.26 & 0.26 \\
\hline SCH Confinement Factor & 0.56 & 0.56 & 0.56 \\
\hline Optical Coupling Efficiency $(O C E)$ & 1.00 & 0.60 & 0.60 \\
\hline Linear Gain Coefficient $\left(G_{\text {coeff }}\right)$ & $3 \cdot 10^{-20} \mathrm{~m}^{2}$ & $4.63 \cdot 10^{-20} \mathrm{~m}^{2}$ & $4.63 \cdot 10^{-20} \mathrm{~m}^{2}$ \\
\hline Carrier Density Reference Gain & $2 \cdot 10^{24} \mathrm{~m}^{-3}$ & $1 \cdot 10^{24} \mathrm{~m}^{-3}$ & $1 \cdot 10^{24} \mathrm{~m}^{-3}$ \\
\hline Carrier Density in the Transparency & $1.5 \cdot 10^{24} \mathrm{~m}^{-3}$ & $0.91 \cdot 10^{24} \mathrm{~m}^{-3}$ & $0.911 \cdot 10^{24} \mathrm{~m}^{-3}$ \\
\hline Initial Carrier Density $(I C D)$ & $1 \cdot 10^{24} \mathrm{~m}^{-3}$ & $1 \cdot 10^{23} \mathrm{~m}^{-3}$ & $1 \cdot 10^{23} \mathrm{~m}^{-3}$ \\
\hline Nonlinear Gain Coefficient $(\varepsilon)$ & $1 \cdot 10^{-23} \mathrm{~m}^{3}$ & $1 \cdot 10^{-21} \mathrm{~m}^{3}$ & $2.9 \cdot 10^{-21} \mathrm{~m}^{3}$ \\
\hline Linear Recombination $\left(A_{\text {recomb }}\right)$ & $1 \cdot 10^{7} \mathrm{~s}^{-1}$ & $5.5 \cdot 10^{8} \mathrm{~s}^{-1}$ & $5.5 \cdot 10^{8} \mathrm{~s}^{-1}$ \\
\hline Bimolecular Recombination $(B)$ & $1 \cdot 10^{-16} \mathrm{~m}^{3} \cdot \mathrm{s}^{-1}$ & $2 \cdot 10^{-16} \mathrm{~m}^{3} \cdot \mathrm{s}^{-1}$ & $2 \cdot 10^{-17} \mathrm{~m}^{3} \cdot \mathrm{s}^{-1}$ \\
\hline Auger recombination $\left(C_{\text {recomb }}\right)$ & $1.3 \cdot 10^{-41} \mathrm{~m}^{6} \cdot \mathrm{s}^{-1}$ & $0.5 \cdot 10^{-41} \mathrm{~m}^{6} \cdot \mathrm{s}^{-1}$ & $0.5 \cdot 10^{-41} \mathrm{~m}^{6} \cdot \mathrm{s}^{-1}$ \\
\hline Carrier Capture Time Constant & $7 \cdot 10^{-11} \mathrm{~s}$ & $7 \cdot 10^{-12} \mathrm{~s}$ & $7 \cdot 10^{-12} \mathrm{~s}$ \\
\hline Carrier Escape Time Constant & $14 \cdot 10^{-11} \mathrm{~s}$ & $14 \cdot 10^{-11} \mathrm{~s}$ & $14 \cdot 10^{-11} \mathrm{~s}$ \\
\hline
\end{tabular}

This approach was experimentally implemented by using the setup presented in Fig. 4, with the electrical current injected into the SOA heterojunction being formed only by the direct current bias component (i.e., switching pulses equal to zero). The laser was in continuous wave (CW) mode at $1550 \mathrm{~nm}$ and an optical isolator in its output prevented optical backward propagation. The optical carrier was then amplified by the SOA with its output acquired by an optical spectrum analyser (OSA). By changing the SOA bias current (from $40 \mathrm{~mA}$ up to $180 \mathrm{~mA}$ ) and input optical power (from $-25 \mathrm{dBm}$ up to $8 \mathrm{dBm}$ ), it was possible to characterize the SOA static optical gain behavior, which was the reference for the VPI model calibration, as presented in Fig. 5 and 6.

The VPI SOA model uses the transmission-line matrix (TLM) method to emulate the propagation of light across the device active region. Its static calibration begins with the SOA active region length experimental evaluation via [48], which is then set in the VPI model. Next, the parameters listed in Table 2 were individually tuned from its default values, analysing the impact of their variation on the simulated curves of optical gain versus bias current and versus input optical power. 'Default' are values preconfigured for a standard SOA in the VPI software and that were used as start profile for the calibration technique presented in [47]. The SOA active region's optical confinement factor, width and thickness are parameters of greater interest and impact on the overall static behavior and, then, firstly analysed. The relationship among these three parameters also determines the saturation power of the SOA output [40]. To analyse the joint relationship among them, a simultaneous scan of the three parameters was performed, resulting in the initial SOA gain curve calibration presented in Fig. 
5(a). Following, parameters with less impact were individually adjusted, allowing fine tuning of the SOA saturation and gain, as presented in Fig. 5(b). A final model calibration was performed through the analysis of the SOA optical gain as a function of its input optical power, as presented in Fig. 6 . The calibrated parameters, which resulted in the behavior of Fig. 5(b) and Fig. 6 and are presented in Table 2 in the "Static" column, are then used in the SOA rate equations solving and applied to the wave equations, which are used to model the optical signal propagation within the active region of the device. The solutions of these equations are obtained numerically by the TLM method [49].

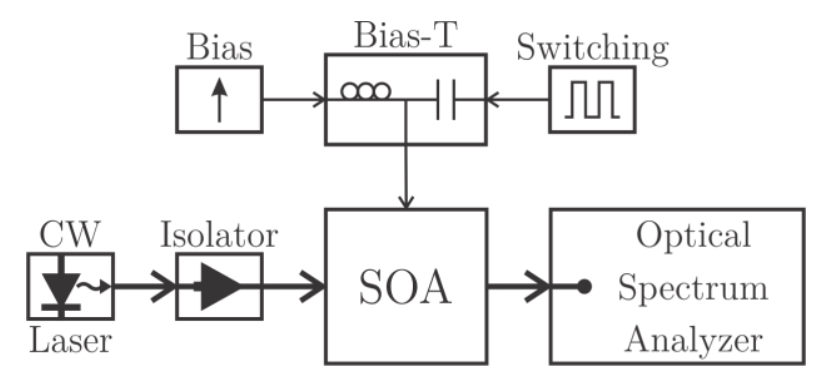

Fig. 4: Experimental setup for the SOA static gain characterization employed as reference for the VPI model calibration.

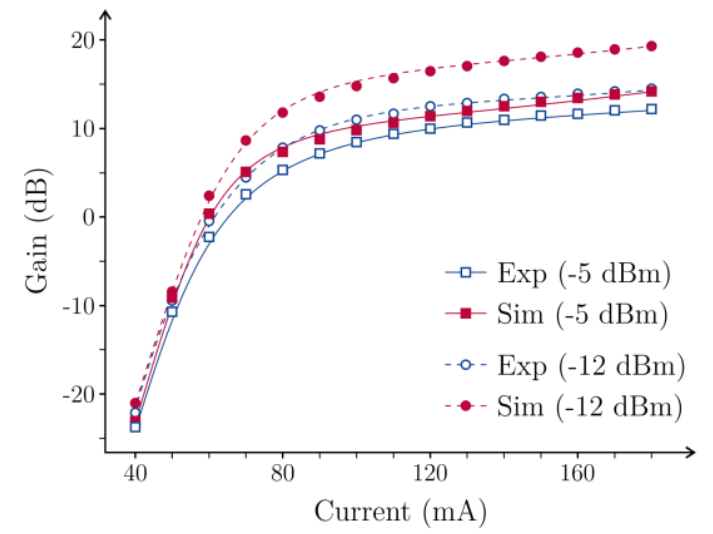

(a)

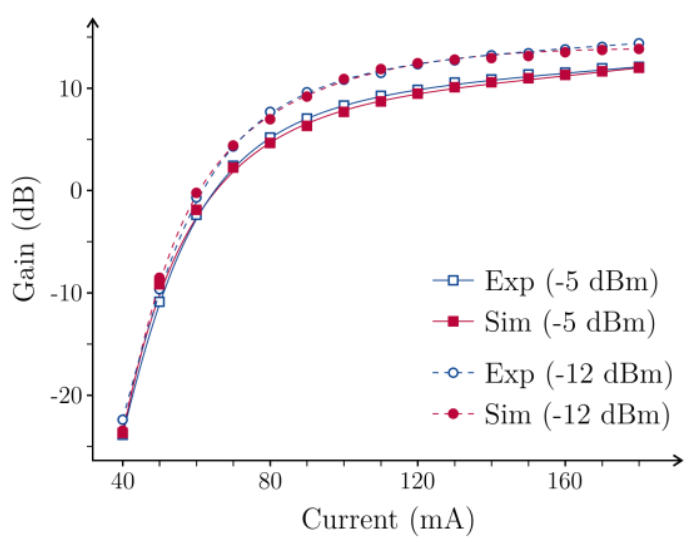

(b)

Fig. 5: Gain versus bias current for simulated and experimental data: (a) preliminary fitting considering only the VPI SOA model active region confinement factor, width, and thickness; (b) fine fitting considering all VPI SOA model parameters. 


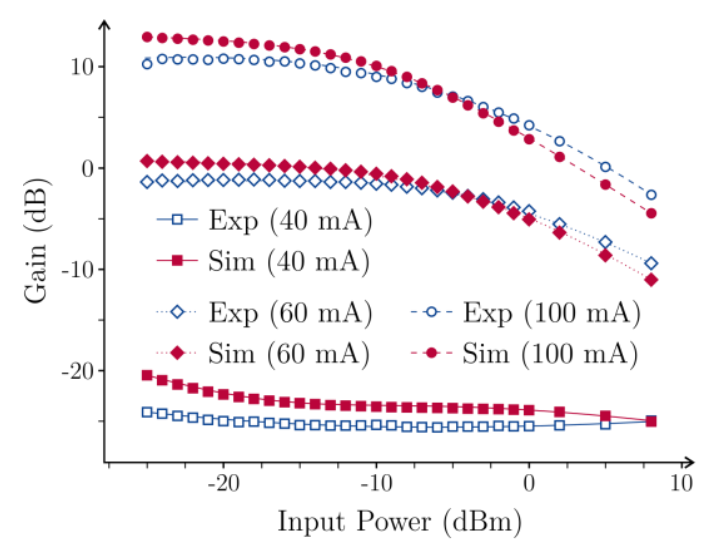

(a)

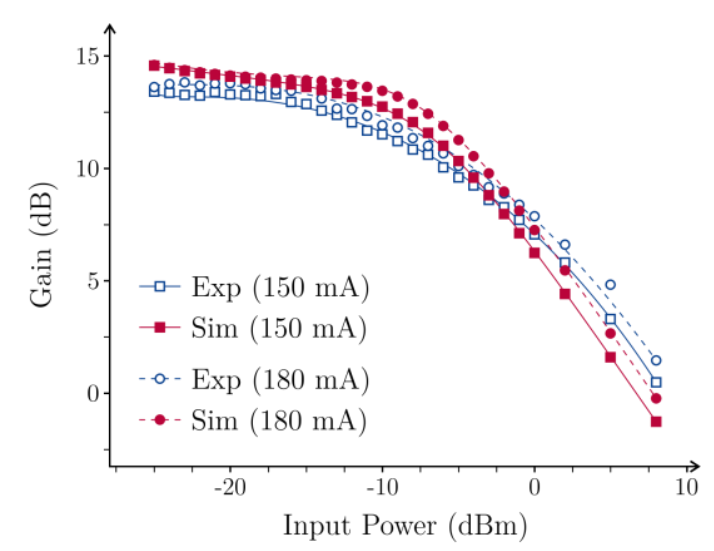

(b)

Figure 6: Gain versus input optical power for simulated and experimental data: (a) SOA bias current equal to $40 \mathrm{~mA}, 60 \mathrm{~mA}$, and $100 \mathrm{~mA}$; (b) SOA bias current equal to $150 \mathrm{~mA}$ and 180 mA.

\section{Dynamic response calibration}

The static gain calibration performed in the previous section does not necessarily describes the SOA dynamic behavior when operating as an electro-optical switch. To precisely evaluate the influence of each SOA model parameter in its dynamic operation, it is fundamental to realize the calibration employing experimental data for time-domain switching pulses as reference, such as the one presented in Fig. 3. However, as the VPI model does not include the SOA microwave mounting responsible for injecting the switching pulses into the SOA, the equivalent circuit of Section A was employed to evaluate the impact of the SOA encapsulation parasitic elements over the device overall performance. In this sense, the setup of Fig. 4 was used with the electrical current injected into the SOA being the sum of its bias and PISIC [50] switching pulses evaluated via the Simulink equivalent circuit. From the SOA VPI model parameters calibrated in the previously section, additional tuning was heuristically performed to approximate the simulated optical pulses to the experimental ones. An initial calibration was performed by considering the SOA operating with a $100 \mathrm{~mA}$ bias current plus an alternate switching pulse formed by a $3 \mathrm{~V}$ step without pre-impulse (directly applied to the SOA using a $47 \Omega$ resistor in series with the SOA input impedance). After that, the resulting model was evaluated for the SOA operating under several conditions (bias current from 100 to $200 \mathrm{~mA}$, step amplitude from 1 to $3 \mathrm{~V}$, and 0:64 ns pre-impulse from 0 to $3 \mathrm{~V}$ ), as presented in Fig. 7. The obtained calibrated parameters to accurately model the SOA dynamic behavior are presented in the "Dynamic" column of Table 2.

\section{RESULTS AND DISCUSSIONS}

The CIP-NL modelling in the VPI environment can be verified by the time domain optical pulses presented in Fig. 7 and by the evaluation metrics of Table 3, which describes quantitatively the SOA simulation switching pulse precision in comparison with the experimental reference absolute values. It 
is possible to note in Fig. 7 that, by comparing to the SOA switching current injected into the SOA heterojunction (previously evaluated through the Simulink equivalent circuit calibration), the VPI model was able to approximately emulate the SOA complex dynamic between optical and electrical carriers in its active region, matching the SOA transient behaviour, both regarding the oscillation amplitude and, more important, frequency. As the SOA oscillations are directly linked to the coupled dependence between carrier density and refraction index in its cavity, small variations on the model calibration can lead to a significant impact in this complex simulation. Furthermore, each switching pulse presented in Fig. 7 represents the SOA operating under different conditions, including variations in its bias current, switching step amplitude, and PISIC pre-impulse amplitude. Each one of these operational parameters has a significant influence in the SOA dynamic behavior and is directly linked with its optical gain and nonlinear effects, proving that the VPI model became able to accurately emulate the actual SOA performance in real operational conditions.

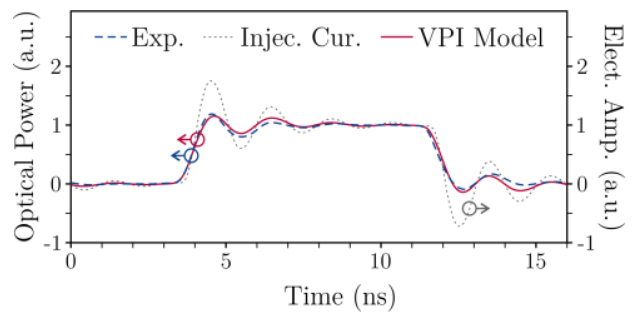

(a)

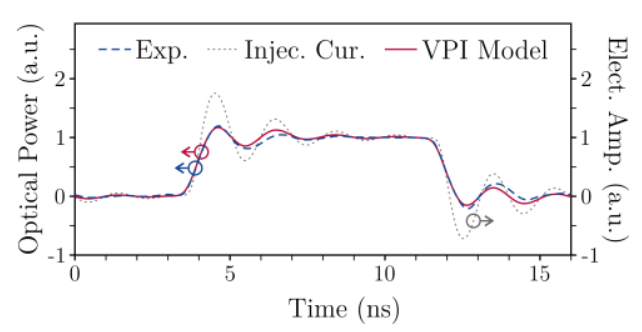

(c)

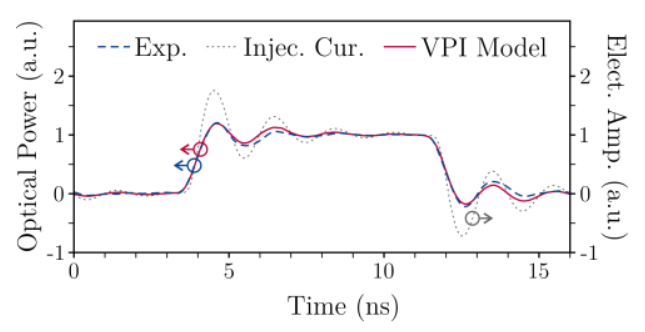

(e)

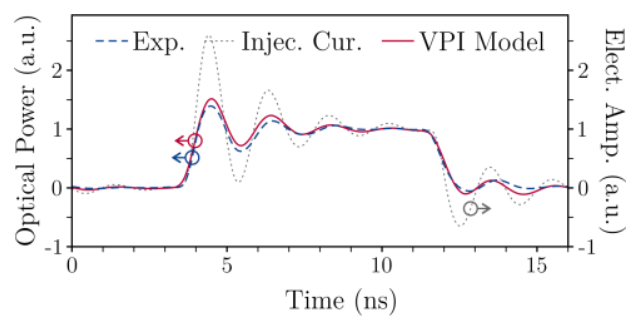

(b)

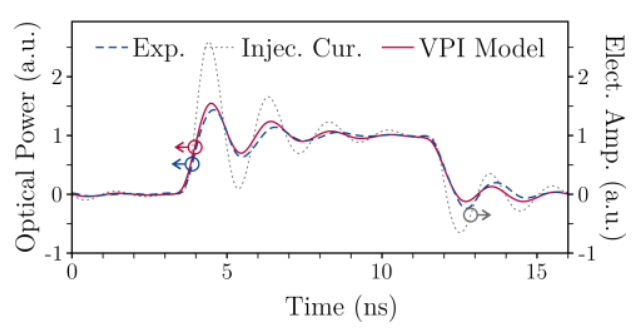

(d)

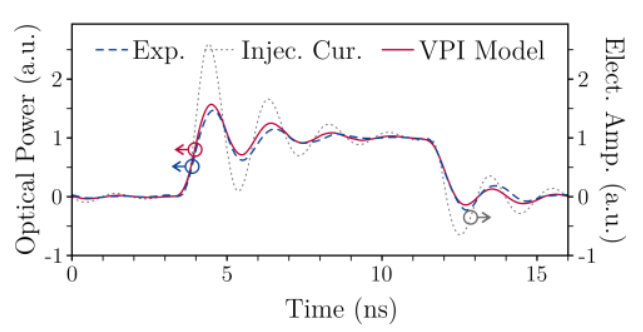

(f)

Fig. 7: Simulated injected current in the SOA CIP-NL and comparison between time-domain optical switching pulses from the calibrated VPI SOA model and the experimental references under several operational conditions: (a) bias current equal to $100 \mathrm{~mA}$, switching step amplitude equal to $2 \mathrm{~V}$, and PISIC pre-impulse amplitude equal to $0 \mathrm{~V}$; (b) bias equal to 100 $\mathrm{mA}$, step equal to $3 \mathrm{~V}$, and pre-impulse equal to $3 \mathrm{~V}$; (c) bias equal to $150 \mathrm{~mA}$, step equal to $2 \mathrm{~V}$, and pre-impulse equal to $0 \mathrm{~V}$; (d) bias equal to $150 \mathrm{~mA}$, step equal to $3 \mathrm{~V}$, and pre-impulse equal to $3 \mathrm{~V}$; (e) bias equal to $200 \mathrm{~mA}$, step equal to $2 \mathrm{~V}$, and pre-impulse equal to $0 \mathrm{~V}$; (f) bias equal to $200 \mathrm{~mA}$, step equal to $3 \mathrm{~V}$, and pre-impulse equal to $3 \mathrm{~V}$. 
A more quantitatively analysis is possible in Table 3, where the most important switching metrics (i.e., rise time, overshoot, and stabilization time) are compared between the experimental reference and the simulated VPI model. Note that the VPI model has a better correspondence with the experimental pulses when operating in a intermediary bias current. In this region, the SOA operates away from its deep nonlinear behavior. However, in all cases the errors in the evaluated metrics are relatively low in comparison with the experimental references absolute values, validating the model technique here proposed.

TABLE III. COMPARISON BETWEEN THE CALIBRATED VPI SOA MODEL AND THE EXPERIMENTAL REFERENCES.

\begin{tabular}{|c|c|c|c|c|c|c|c|c|}
\hline \multirow[b]{2}{*}{$\begin{array}{c}\text { SOA Bias } \\
\text { Current }\end{array}$} & \multirow{2}{*}{$\begin{array}{c}\text { Switching } \\
\text { Step } \\
\text { Amplitude }\end{array}$} & \multirow{2}{*}{$\begin{array}{c}\text { PISIC } \\
\text { Pre- } \\
\text { impulse } \\
\text { Amnlitude } \\
\end{array}$} & \multicolumn{2}{|c|}{ Rise Time } & \multicolumn{2}{|c|}{ Overshoot } & \multicolumn{2}{|c|}{ Stabilization Time } \\
\hline & & & $\begin{array}{l}\text { Exp. } \\
\text { Ref. }\end{array}$ & $\begin{array}{l}\text { Model } \\
\text { Error } \\
\end{array}$ & $\begin{array}{l}\text { Exp. } \\
\text { Ref. }\end{array}$ & $\begin{array}{l}\text { Model } \\
\text { Error }\end{array}$ & $\begin{array}{l}\text { Exp. } \\
\text { Ref. }\end{array}$ & $\begin{array}{l}\text { Model } \\
\text { Error }\end{array}$ \\
\hline $100 \mathrm{~mA}$ & 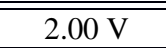 & $0.00 \mathrm{~V}$ & $5650.62 \mathrm{ps}$ & $8.6 \%$ & $19.11 \%$ & $3.31 \%$ & 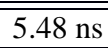 & $60.94 \mathrm{ps}$ \\
\hline $100 \mathrm{~mA}$ & $3.00 \mathrm{~V}$ & $3.00 \mathrm{~V}$ & $401.32 \mathrm{ps}$ & $16.74 \%$ & $39.07 \%$ & $12.43 \%$ & $5.42 \mathrm{~ns}$ & $92.19 \mathrm{ps}$ \\
\hline $150 \mathrm{~mA}$ & $2.00 \mathrm{~V}$ & $0.00 \mathrm{~V}$ & $621.88 \mathrm{ps}$ & $2.26 \%$ & $19.57 \%$ & $2.48 \%$ & $5.54 \mathrm{~ns}$ & $7.81 \mathrm{ps}$ \\
\hline $150 \mathrm{~mA}$ & $3.00 \mathrm{~V}$ & $3.00 \mathrm{~V}$ & $443.75 \mathrm{ps}$ & $3.87 \%$ & $44.03 \%$ & $10.97 \%$ & $5.51 \mathrm{~ns}$ & $7.92 \mathrm{ps}$ \\
\hline $200 \mathrm{~mA}$ & $2.00 \mathrm{~V}$ & $0.00 \mathrm{~V}$ & $629.29 \mathrm{ps}$ & $3.23 \%$ & $20.77 \%$ & $1.29 \%$ & $5.54 \mathrm{~ns}$ & $1.57 \mathrm{ps}$ \\
\hline $200 \mathrm{~mA}$ & $3.00 \mathrm{~V}$ & $3.00 \mathrm{~V}$ & $431.25 \mathrm{ps}$ & $2.17 \%$ & $47.04 \%$ & $10.11 \%$ & $5.48 \mathrm{~ns}$ & $10.01 \mathrm{ps}$ \\
\hline
\end{tabular}

\section{CONCLUSION}

The SOA is a promising electro-optical component, able to amplify, switch, regenerate, and route an optical carrier. Yet, several of these applications are influenced or based on its highly nonlinear behavior, which demands a careful device characterization and modelling to adequate its operation to the desired application requirements. As the SOA performance is intrinsically dependent with its complex active region carrier dynamics, each of its constructive and mounting parameters have a significant impact on the model accuracy, turning the default model available in simulation softwares unsuitable for the evaluation of the device impact on sophisticated optical networks.

The work here presented proposes a modeling technique capable of systematically predicting the electrical current injected into the SOA heterojunction, considering the overall degradation imposed by parasitic elements from the device mounting and encapsulation, by using an automated calibration of an electrical equivalent circuit. Although this approach is capable of providing an initial good approximation for the SOA optical output, the precise emulation of its nonlinear behavior demands a more complex model to evaluate SOA active region carrier dynamics and its influence in the SOA refractive index. To do that, the SOA injected current estimated by the equivalent circuit is employed as the input electrical signal for the SOA VPI default model, which in turn is carefully tuned to precisely emulate the SOA electro-optical performance, including its transient behavior. The results showed a good approximation between the calibrated VPI model and the experimental reference, including the precise reproduction of the SOA dynamic switching. An accurate model, as the one 
proposed here, can be crucial during the design of sophisticated devices and its application on complex and efficient optical networks.

\section{ACKNOWLEDGMENT}

The authors would like to thank Prof. Dr. Adriano L. Toazza, Dr. Napoleão S. Ribeiro, and Dr. Rafael C. Figueiredo for their contributions to previously stages of this work. This work was supported in part by the Brazilian agencies São Paulo Research Foundation (FAPESP - grants 2017/20121-8, 2015/50063-4, 2015/24517-8, and 2007/56024-4) and National Council for Scientific and Technological Development (CNPq - grants 301409/2017-0, 159388/2017-1, 400129/2017-5, 402923/2016-2, and 301420/2015-7.

\section{REFERENCES}

[1] H. Meyer, J. C. Sancho, M. Mrdakovic, W. Miao, N. Calabretta, Optical packet switching in HPC: An analysis of applications performance, Future Generation Computer Systems doi:10.1016/j.future.2017.02.041.

[2] B. D. Feris, P. Gravey, P. Morel, M. L. Moulinard, M. Morvan, A. Sharaiha, Dimensioning of 112G optical-packetswitching-based interconnects for energy-efficient data centers, IEEE/OSA Journal of Optical Communications and Networking 9 (4) (2017) B124-B136. doi:10.1364/JOCN.9.00B124.

[3] S. Ni, B. Wu, Y. Liu, Characteristics of silicon-based Sagnac optical switches using magneto-optical micro-ring array, Fiber and Integrated Optics 37 (1) (2018) 12-20. doi:10.1080/01468030.2017.1423135.

[4] J. Renaudier, A. C. Meseguer, A. Ghazisaeidi, P. Tran, R. R. Muller, Brenot, A. Verdier, F. Blache, K. Mekhazni, B. Duval, H. Debregeas, M. Achouche, A. Boutin, F. Morin, L. Letteron, N. Fontaine, Y. Frignac, G. Charlet, First 100$\mathrm{nm}$ continuous-band WDM transmission system with $115 \mathrm{~Tb} / \mathrm{s}$ transport over $100 \mathrm{~km}$ using novel ultra-wideband semiconductor optical amplifiers, in: Optical Communication, 2017. 43rd European Conference on, Vol. 1, 2017, pp. 13.

[5] Y. M. Zhang, Y. Liu, Z. K. Zhang, Z. P. Zhao, Y. Tian, N. H. Zhu, Reconfigurable WDM-PON empowered by a lowcost 8-channel directly modulated laser module, Optoelectronics Letters 13 (6) (2017) 423-426. doi:10.1007/s11801017-7159-6.

[6] C. Zhang, T. Ning, J. Li, C. Li, X. He, L. Pei, Simultaneous MMW generation and up-conversion for WDM-ROF systems based on FP laser, Optics \& Laser Technology 84 (2016) 94 - 101. doi:10.1016/j.optlastec.2016. 05.010.

[7] T. Durhuus, B. Mikkelsen, C. Joergensen, S. L. Danielsen, K. E. Stubkjaer, All-optical wavelength conversion by semiconductor optical amplifiers, Journal of Lightwave Technology 14 (6) (1996) 942-954. doi: 10.1109/50.511594.

[8] F. Saadaoui, M. Fathallah, A. M. Ragheb, M. I. Memon, H. Fathallah, S. A. Alshebeili, Optimizing OSSB generation using semiconductor optical amplifier (SOA) for 5G millimeter wave switching, IEEE Access 5 (2017) 6715-6723. doi:10.1109/ACCESS.2017.2683064.

[9] Z. Shen, L. Wang, X. Wang, Y. Cao, X. Feng, B. ou Guan, Tunable dual wavelength single-longitudinal-mode fiber laser based on spectral narrow-ing effect in a nonlinear semiconductor optical amplifier, Optics \& Laser Technology 94 (2017) 72 - 76. doi:10.1016/j.optlastec.2017.03.020.

[10] X. Zheng, O. Raz, N. Calabretta, D. Zhao, R. Lu, Y. Liu, Multiport InP monolithically integrated all-optical wavelength router, Optics Letters 41 (16) (2016) 3892-3895. doi:10.1364/OL.41.003892.

[11] A. Chiuchiarelli, C. M. Gallep, E. Conforti, Fabry Perot laser-based optical switch for multicast transmission in bidirectional optical access networks, Microwave and Optical Technology Letters 58 (6) (2016) 1466-1469. doi: 10.1002/mop.29823.

[12] N. S. Ribeiro, C. M. Gallep, E. Conforti, Semiconductor optical amplifier cavity length impact over data erasing/rewriting, Microwave and Optical Technology Letters 55 (5) (2013) 998-1001. doi:10.1002/mop.27496.

[13] A. Kotb, K. E. Zoiros, Performance analysis of all-optical XOR gate with photonic crystal semiconductor optical amplifier-assisted MachZehnder interferometer at $160 \mathrm{~Gb} / \mathrm{s}$, Optics Communications 402 (2017) 511-517. doi:10.1016/j.optcom.2017.06.050.

[14] E. Dimitriadou, K. Zoiros, Proposal for ultrafast all-optical XNOR gate using single quantum-dot semiconductor optical amplifier-based Mach Zehnder interferometer, Optics \& Laser Technology 45 (2013) 79 - 88. doi: 10.1016/j.optlastec.2012.07.024.

[15] D. Mandal, S. Mandal, S. K. Garai, Alternative approach of developing all-optical Fredkin and Toffoli gates, Optics \& Laser Technology 72 (2015) 33 - 41. doi:10.1016/j.optlastec.2015.03.010. 
[16] S. Mandal, D. Mandal, M. K. Mandal, S. K. Garai, Design of optical quaternary adder and subtractor using polarization switching, Journal of Optics. doi:10.1007/s12596-018-0460-3.

[17] D. Brunina, D. Liu, K. Bergman, An energy-efficient optically connected memory module for hybrid packet- and circuit-switched optical networks, IEEE Journal of Selected Topics in Quantum Electronics 19 (2). doi: 10.1109/JSTQE.2012.2224096.

[18] T. Tanemura, I. M. Soganci, T. Oyama, T. Ohyama, S. Mino, K. A. Williams, N. Calabretta, H. J. S. Dorren, Y. Nakano, Large-capacity compact optical buffer based on InP integrated phased-array switch and coiled fiber delay lines, Journal of Lightwave Technology 29 (4) (2011) 396-402. doi:10.1109/JLT.2010.2102338.

[19] G. Berrettini, G. Meloni, L. Poti, A. Bogoni, All-optical variable buffer based on semiconductor optical amplifier, IEEE Journal of Quantum Electronics 47 (4) (2011) 510-516. doi:10.1109/JQE.2010.2092752.

[20] W. Yongjun, X. Xiangjun, S. Chao, Multichannel packets buffering in cascaded double-loop optical buffer based on the pure phase switching of gain-transparent semiconductor optical amplifier, Optical Engineering 53. doi:10.1117/1.OE.53.1.016101.

[21] M. Matsuura, F. Gomez-Agis, N. Calabretta, O. Raz, H. J. S. Dorren, 320to-40-Gb/s optical demultiplexing using fourwave mixing in a quantum dot SOA, IEEE Photonics Technology Letters 24 (2) (2012) 101-103. doi: 10.1109/LPT.2011.2173672.

[22] R. Stabile, N. Calabretta, K. A. Williams, Switch-filter wavelength selector: simulation and experiment, IET Optoelectronics 8 (1) (2014) 1-10. doi: 10.1049/iet-opt.2013.0084.

[23] G. Chen, L. Xi, Y. Ma, L. Sun, X. Zhang, Regeneration of DQPSK signals using semiconductor optical amplifier-based phase regenerator, in: International Conference on Advanced Infocom Technology 2011 (ICAIT 2011), 2011. doi:10.1049/cp.2011.1081.

[24] G. Gavioli, P. Bayvel, Novel 3R regenerator based on polarization switching in a semiconductor optical amplifierassisted fiber Sagnac interferometer, IEEE Photonics Technology Letters 15 (9) (2003) 1261-1263. doi:10. 1109/LPT.2003.816700.

[25] P. Vorreau, A. Marculescu, J. Wang, G. Bottger, B. Sartorius, C. Bornholdt, J. Slovak, M. Schlak, C. Schmidt, S. Tsadka, W. Freude, J. Leuthold, Cascadability and regenerative properties of SOA all-optical DPSK wavelength converters, IEEE Photonics Technology Letters 18 (18) (2006) 1970-1972. doi:10.1109/LPT.2006.880714.

[26] Y. Zhan, M. Zhang, M. Liu, L. Liu, X. Chen, All-optical QPSK signal regeneration based on XPM in semiconductor optical amplifier, in: 2012 Asia Communications and Photonics Conference (ACP), 2012, pp. 1-3.

[27] P. Rocha, C. M. Gallep, E. Conforti, All-optical mitigation of amplitude and phase-shift drift noise in semiconductor optical amplifiers, Optical Engineering 54. doi:10.1117/1.OE.54.10.106110.

[28] A. Shacham, K. Bergman, An experimental validation of a wavelength striped, packet switched, optical interconnection network, Journal of Lightwave Technology 27 (7) (2009) 841-850. doi:10.1109/JLT.2008.928541.

[29] A. Farmani, M. Farhang, M. H. Sheikhi, High performance polarization independent quantum dot semiconductor optical amplifier with $22 \mathrm{~dB}$ fiber to fiber gain using mode propagation tuning without additional polarization controller, Optics \& Laser Technology 93 (2017) 127 - 132. doi:10.1016/j.optlastec.2017.02.007.

[30] R. C. Figueiredo, N. S. Ribeiro, A. M. O. Ribeiro, C. M. Gallep, E. Conforti, Hundred-picoseconds electro-optical switching with semiconductor optical amplifiers using multi-impulse step injection current, Journal of Lightwave Technology 33 (1) (2015) 69-77. doi:10.1109/JLT.2014.2372893.

[31] A. Ehrhardt, M. Eiselt, G. Grossopf, L. Kuller, R. Ludwig, W. Pieper, Schnabel, H. G. Weber, Semiconductor laser amplifier as optical switch-ing gate, Journal of Lightwave Technology 11 (8) (1993) 1287-1295. doi: 10.1109/50.254087.

[32] M. Renaud, M. Bachmann, M. Erman, Semiconductor optical space switches, IEEE Journal of Selected Topics in Quantum Electronics 2 (2) (1996) 277-288. doi:10.1109/2944.577378.

[33] R. C. Figueiredo, T. Sutili, N. S. Ribeiro, C. M. Gallep, E. Conforti, Semiconductor optical amplifier space switch with symmetrical thin-film resistive current injection, Journal of Lightwave Technology 35 (2) (2017) $280-287$. doi:10.1109/JLT.2016.2635202.

[34] H. Wang, E. T. Aw, K. A. Williams, A. Wonfor, R. V. Penty, I. H. White, Lossless multistage SOA switch fabric using high capacity monolithic 4x4 SOA circuits, in: 2009 Conference on Optical Fiber Communication, 2009, pp. 1-3.

[35] N. S. Ribeiro, A. L. Toazza, C. M. Gallep, E. Conforti, Rise time and gain fluctuations of an electro-optical amplified switch based on multipulse injection in semiconductor optical amplifiers, IEEE Photonics Technology Letters 21 (12) (2009) 769-771. doi:10.1109/LPT.2009.2017731. 
Journal of Microwaves, Optoelectronics and Electromagnetic Applications, Vol. 17, No. 4, December 2018 DOI: http://dx.doi.org/10.1590/2179-10742018v17i41404

[36] R. C. Figueiredo, E. C. Magalhes, N. S. Ribeiro, C. M. Gallep, E. Conforti, Equivalent circuit of a semiconductor optical amplifier chip with the bias current influence, in: 2011 SBMO/IEEE MTT-S International Microwave and Optoelectronics Conference (IMOC 2011), 2011, pp. 852-856. doi: 10.1109/IMOC.2011.6169263.

[37] I. Sengupta, A. Das Barman, Investigation of inter-channel crosstalk mitigation by assist light in a toad switch by using electrical equivalent circuit model of soa, Optical and Quantum Electronics 47 (2) (2015) 331-350. doi:10.1007/s11082014-9916-0.

[38] W. Mathlouthi, P. Lemieux, M. Salsi, A. Vannucci, A. Bononi, L. A. Rusch, Fast and efficient dynamic wdm semiconductor optical amplifier model, Journal of Lightwave Technology 24 (11) (2006) 4353-4365. doi:10.1109/JLT.2006.884217.

[39] M. J. Connelly, Wideband semiconductor optical amplifier steady-state numerical model, IEEE Journal of Quantum Electronics 37 (3) (2001) 4390-447. doi:10.1109/3.910455.

[40] A. D. Barman, M. Scaffardi, S. Debnath, L. Pot, A. Bogoni, Design tool and its experimental validation for soa-based photonic signal processing, Optical Fiber Technology 15 (1) (2009) 39-49. doi:10.1016/j.yofte.2008.04.002.

[41] Mathworks Simulink, [www.mathworks.com/products/simulink.html].

[42] VPIphotonics, [http://www.vpiphotonics.com/index.php].

[43] R. Tucker, D. Pope, Circuit modeling of the effect of diffusion on damping in a narrow-stripe semiconductor laser, IEEE Journal of Quantum Electronics19 (7) (1983) 1179-1183. doi:10.1109/JQE.1983.1072005.

[44] R. C. Figueiredo, N. S. Ribeiro, C. de Mello Gallep, E. Conforti, Frequency and time-domain simulations of semiconductor optical amplifiers using equivalent circuit modeling, Optical Engineering 54. doi:10.1117/1.OE. 54.11.114107.

[45] R. C. Figueiredo, N. S. Ribeiro, C. M. Gallep, E. Conforti, Bias current influence on semiconductor optical amplifier's equivalent circuit, Optics Communications 336 (2015) 153 - 159. doi:10.1016/j.optcom.2014.09. 069.

[46] R. C. Figueiredo, E. C. Magalhes, N. S. Ribeiro, C. M. Gallep, E. Conforti, Equivalent circuit of a semiconductor optical amplifier chip with the bias current influence, in: 2011 SBMO/IEEE MTT-S International Microwave and Optoelectronics Conference (IMOC 2011), 2011, pp. 852-856. doi: 10.1109/IMOC.2011.6169263.

[47] P. Rocha, C. M. Gallep, T. Sutili, E. Conforti, Calibration of TLM model for semiconductor optical amplifier by heuristic parameters' extraction", Journal of Microwaves, Optoelectronics and Electromagnetic Applications, vol. 17, $\mathrm{n}^{\circ}$ 2, pp. 260-267, 2018.

[48] S. M. Sze, K. K. Ng, Physics of Semiconductor Devices, 3rd Edition, Wiley Inter science publication, John Wiley \& Sons, 2007.

[49] VPI Transmission Maker, Photonics circuits users manual, VPI photonics.

[50] C. M. Gallep, E. Conforti, Reduction of semiconductor optical amplifier switching times by pre-impulse step-injected current technique, IEEE Photonics Technology Letters 14 (7) (2002) 902-904. doi:10.1109/LPT.2002.1012379. 